\title{
Correlates of sitting time in adults with type 2 diabetes
}

\author{
Anne-Sophie Brazeau, Samantha Hajna, Lawrence Joseph and Kaberi Dasgupta*
}

\begin{abstract}
Background: Studies suggest a relationship between sitting time and cardiovascular disease mortality. Our aim was to identify socio-demographic, contextual, and clinical (e.g., body composition, diabetes duration) correlates of self-reported sitting time among adults with type 2 diabetes, a clinical population at high risk for cardiovascular disease. We sought to determine if there was an inverse relationship between sitting and step counts in a diabetes cohort in whom we had previously identified low step counts with further lowering in fall/winter.
\end{abstract}

Methods: The cohort included 198 adults (54 \% men; age 60.0 SD 11.5 years; Body mass index 30.4 SD 5.6 kg/m²) (Montréal, Canada). Socio-demographic, contextual and clinical factors were assessed using standardized questionnaires and step counts with a pedometer over 14 days (concealed viewing windows). Total sitting time was estimated once per season (up to 4 times per year at -month intervals) using the International Physical Activity Questionnaire-Short version. Potential sitting time correlates were evaluated using Bayesian longitudinal hierarchical linear regression models in participants with sitting time data $(n=191)$.

Results: The average sitting time was 308 (SD 161) minutes/day without variation across seasons. Sitting time correlates were being an immigrant (56 fewer minutes/day spent sitting compared to non-immigrants, $95 \%$ credible interval, Crl: $-100,-11)$ and having a university degree (55 more minutes/day spent sitting compared to those without a university degree, $95 \% \mathrm{Crl}: 10,100)$ after adjustment for potential correlates observed in univariate analyses (sex, age, job status, waist circumference, depressed mood, steps). Correlation between sitting and steps, adjusted for age and sex, was -0.144 (95\% Cl: $-0.280,0.002$ ).

Conclusion: There was low correlation between sitting time and step counts. Therefore, high sitting time and low step counts are behaviours that may need to be independently targeted. Interventions to reduce sitting time in adults with type 2 diabetes may need to target non-immigrants and those with a university degree.

Keywords: Sedentary behaviors, Seasons, Steps, Socio-demographic factors

\section{Background}

Greater use of modern technology and electronic entertainment has contributed to reductions in physical movement [1]. Adults with established type 2 diabetes are characterized by low levels of physical activity with step counts falling in the low active category according to the cut-offs proposed by Tudor-Locke and Bassett $[2,3]$. We have previously determined pedometerassessed daily step counts to average 5365 steps/day (Standard deviation, $\mathrm{SD}=2655$ ) in this clinical population, with higher step counts during the spring/summer seasons

\footnotetext{
* Correspondence: kaberi.dasgupta@mcgill.ca

Department of Medicine, McGill University, 687 Pine Avenue West, V-Building (V0.06), Montréal, QC H3A 1A1, Canada
}

compared to the fall/winter seasons [4]. We have also determined higher step counts to be associated with lower blood pressure [5], A1C, adiposity measures [6], consistent with the impact of pedometer-based interventions [7] and a recent longitudinal analysis demonstrating the effects of higher step counts on reductions in cardiovascular mortality [8].

While increasing step counts is clearly an imperative in adults with type 2 diabetes, an area that is emerging as an additional issue is sitting time. Sedentary and sitting time have been linked not only with the development of type 2 diabetes and cardiovascular disease [9, 10], but also with both cardiovascular-specific and all-cause mortality (greatest compared to lowest sedentary time pooled hazard ratio 
1.9, $95 \%$ confidence interval (CI) 1.4, 2.7 [9] and $>7 \mathrm{~h} /$ week compared to $<1 \mathrm{~h} /$ day of television watching hazard ratio 1.5, $95 \% \mathrm{CI} 1.1,2.0,[11])$. Although there is between-country variation, average sitting time in adult populations, measured by self-report, is approximately 300 to $360 \mathrm{~min}$ per day (i.e., 5 to $6 \mathrm{~h}$ ) $[12,13]$.

Both high sitting time and low step counts are thus undesirable behaviours for optimal health. It is not clear whether those with low step counts are the same group of individuals with high sitting time. Clarification of this issue would help to strengthen the developing of health behaviour-enhancing interventions aiming to reduce vascular disease risk in type 2 diabetes. To effectively address this newly-emerging vascular risk factor, it would be helpful to identify the sitting time predictors and correlates, particularly in groups at increased risk for mortality, such as people with type 2 diabetes [14].

Therefore, the aims of this study were, in adults with type 2 diabetes, 1) to quantify overall daily sitting time and variation across seasons, 2) to compare sitting and step counts in this clinical population, and 3) to identify the socio-demographic, contextual and health-related factors of self-reported sitting time in this population. We sought to determine if there was an inverse relationship between sitting and pedometer-assessed step counts and if there was seasonal variation in sitting time similar to that previously observed for step counts.

\section{Methods}

This study is a secondary analysis of a prospective cohort study of adults $(n=201)$ with physician-diagnosed type 2 diabetes recruited through McGill University-affiliated outpatient clinics conducted in Montréal (Canada). Following informed consent, participants underwent consecutive seasonal assessments (i.e., 4 assessments) over a oneyear follow-up period between June 2006 and June 2009. Complete description of the protocol can be found elsewhere but important elements are described herein $[4,15]$. Procedures were approved by McGill University's Faculty of Medicine Institutional Review Board and all participating institutions (McGill University Health Centre Research Ethics Board, Research Ethics Committee at the Jewish General Hospital and the Comité d'éthique de la recherche du Centre de santé et de services sociaux de la Montagne). The present analysis included data on 198 participants with at least one self-reported value for sitting (i.e., at one of the 4 assessments).

\section{Outcome measurement}

Sitting time was assessed using the International Physical Activity Questionnaire-Short version (IPAQ-SV) (www.ipaq.ki.se) [16]. Participants were queried on the average time spent sitting during week days (hours and minutes per day, converted to minutes per day). This question included sitting time at work, at home, while doing course work and during leisure time. Examples were provided (e.g., time spent sitting at a desk, sitting to watch television). IPAQ-SV has acceptable reliability and validity for assessing usual sitting time $[17,18]$. A recent validation study among 1751 adults demonstrated a correlation coefficient of 0.46 between the IPAQ-SV sitting question and 7-day accelerometry data (Actigraph GT1M) [19].

\section{Potential correlates examined \\ Step counts}

Perception of walking was evaluated with the IPAQ-SV. Participants were asked 2 questions (during the last 7 days, on how many days did you walk for at least $10 \mathrm{~min}$ at a time and how much time did you usually spend walking on one of those days) and average walking time was computed according to the guidelines on how to process the data [20]. Objective measure of walking was assessed with pedometer counts. Step counts were quantified at each evaluation for a 2-week period [4, 15]. Participants were provided with three pedometers labelled A, B and C (Yamax SW-200, viewing window concealed). They wore one (pedometer A) for one week and a second (pedometer B) for another week during waking hours. They then mailed back all three to the study centre in the stamped and addressed envelope provided. The step counts recorded on the third pedometer (C) served to quantify the counts accumulated during the mailing process. This number was subtracted from the totals on the other two pedometers ( $\mathrm{A}$ minus $\mathrm{C}$ and $\mathrm{B}$ minus $\mathrm{C}$ ). The corrected step counts on pedometers $\mathrm{A}$ and $\mathrm{B}$ were summed and divided by the total number of days pedometers were worn as reported by the participants on a form included in the envelope.

\section{Sociodemographic factors}

Sex, education (university education, yes/no), annual household income $(<\$ 50,000$, yes $/$ no), work status (currently working, yes/no), marital status (married/common-law, yes/no), immigrant status (yes/no), and age (years) were assessed at baseline using a standardized questionnaire. For annual household income participants had to choose from 5 different categories $(<\$ 15,000$; $\$ 15,000$ to $\$ 30,000 ; \$ 30,000$ to $\$ 50,000 ; \$ 50,000$ to $\$ 80,000 ;>\$ 80,000)$ and the two last were collapsed in the present analysis.

\section{Contextual factors}

In this analysis, season was based on corresponding solstice calendar definitions of fall (September 22/23 to December 20/21), winter (December 21/22 to March 19/ 20), spring (March 20/21 to June 19/20), and summer (June 20/21 to September 21/22) in the northern hemisphere. Dog ownership (yes/no) was queried as part of 
the baseline questionnaire. Vehicle access (yes/no) was assessed as part of a follow-up questionnaire that was mailed to all participants in the winter of 2012/2013.

\section{Clinical measures}

Depressed mood (yes/no) was assessed using the Center for Epidemiologic Studies-Depression Scale (CES-D Score 216 ), a validated tool for assessing depressive symptomology [21]. Body mass index (BMI in $\mathrm{kg} / \mathrm{m}^{2}$ ) was calculated based on direct weight and height measurements and waist circumference $(\mathrm{cm})$ was measured midway between the lateral lower ribs and the iliac crests. Insulin use (yes/no) and diabetes duration (years) were self-reported at the baseline visit [15].

\section{Statistical analysis}

Data are presented as mean and SD unless state otherwise. Pearson correlations with $95 \%$ CI were calculated. To compare the fall/winter and the spring/summer seasons, we averaged the data collected within these periods and computed absolute differences. Given the similarity of data from spring and summer and from fall and winter, spring and summer data were collapsed, as were fall and winter data, as in a previous analysis of this cohort [4]. Bayesian longitudinal hierarchical linear regression models with diffuse priors were used to estimate the associations of self-reported sitting time in minutes/day measured over time with step counts and each of the socio-demographic, contextual and clinical factors described above (WinBUGS 1.4.3). A series of models were unadjusted, partially adjusted and fully adjusted for the variables identified $a$ priori as potential sitting time correlates. The fully adjusted final model was based on complete case data at baseline $(n=191)$ and included age, gender, immigrant status, education, waist circumference, job status, steps and absence of depressed mood. These determinants were selected given their important association with sitting time in univariate analyses. The importance of findings was based on examination of point estimates and $95 \%$ credible intervals (95\% CrI), the Bayesian analog of frequentist confidence intervals.

\section{Results}

Average sitting time was $308 \mathrm{~min} /$ day (SD 161) equivalent to approximately five hours per day (Table 1). Fifty-one percent of the participants attended all 4 visits, $23.7 \%$ attended

Table 1 Univariate longitudinal hierarchical linear regression estimates between the predictors of interest and daily sitting time in minutes

\begin{tabular}{|c|c|c|c|}
\hline & & Baseline characteristics $N=198$ & Increment in minutes of daily sitting time $(95 \% \mathrm{Crl})^{\mathrm{b}}$ \\
\hline \multirow[t]{2}{*}{ Average daily sitting time (minutes); } & mean (SD) & $308(161)$ & - \\
\hline & median (IQR) & $278(188,405)$ & - \\
\hline \multicolumn{4}{|l|}{ Socio-demographic factors } \\
\hline \multicolumn{2}{|l|}{ Men; $n(\%)$} & $106(54 \%)$ & $-2.9(-4.9,-0.8)$ \\
\hline \multicolumn{2}{|l|}{ Age (years); mean (SD) } & $60.0(10.5)$ & $41.3(-3.5,85.7)$ \\
\hline \multicolumn{2}{|l|}{ University education; $n$ (\%) } & $78(39 \%)$ & $58.2(12.3,103.6)$ \\
\hline \multicolumn{2}{|l|}{ Currently employed; $n$ (\%) } & $113(57 \%)$ & $86.3(42.5,130.0)$ \\
\hline \multicolumn{2}{|c|}{ Annual household income $\geq \$ 50,000 ; n(\%)^{\mathrm{a}}$} & $77(44 \%)$ & $90.0(43.3,134.9)$ \\
\hline \multicolumn{2}{|l|}{ Married/common-law; $n(\%)^{a}$} & $123(69 \%)$ & $-34.9(-86.3,15.4)$ \\
\hline \multicolumn{2}{|l|}{ Immigrant; $n$ (\%) } & $91(46 \%)$ & $-53.3(-98.2,-10.1)$ \\
\hline \multicolumn{4}{|l|}{ Contextual factors } \\
\hline \multicolumn{2}{|l|}{ Dog ownership; $n$ (\%) } & $31(16 \%)$ & $18.2(-40.9,78.3)$ \\
\hline \multicolumn{2}{|l|}{ Regular vehicle access; $n(\%)^{\mathrm{a}}$} & $70(80 \%)$ & $92.0(6.7,179.2)$ \\
\hline \multicolumn{2}{|l|}{ Steps (steps/day); mean (SD) ${ }^{\mathrm{a}}$} & $5361(2473)$ & $0.001(-0.005,0.008)$ \\
\hline \multicolumn{4}{|l|}{ Clinical factors } \\
\hline \multicolumn{2}{|l|}{ Body mass index $\left(\mathrm{kg} / \mathrm{m}^{2}\right)$; mean (SD) } & $30.4(5.6)$ & $1.3(-2.8,5.4)$ \\
\hline \multicolumn{2}{|l|}{ Waist circumference (cm); mean (SD) } & $102.1(13.2)$ & $2.4(1.1,3.6)$ \\
\hline \multicolumn{2}{|l|}{ Depressed mood; $n(\%)$} & $55(28 \%)$ & $-36.5(-71.5,-2.4)$ \\
\hline \multicolumn{2}{|l|}{ Diabetes duration (years); mean (SD) } & $9.4(8.0)$ & $-2.6(-5.4,0.2)$ \\
\hline \multicolumn{2}{|l|}{ Insulin use; $n(\%)$} & $66(33 \%)$ & - \\
\hline
\end{tabular}

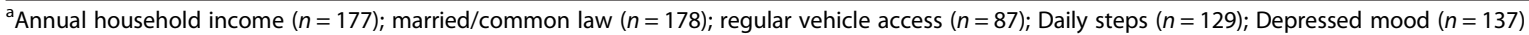

${ }^{\mathrm{b}}$ Modelling independent variables at baseline except for depression and steps/day which were modelled over time; sitting time was modelled over time 
3 visits, $13.6 \%$ attended 2 visits and $11.6 \%$ attended only 1 visit. A plot of reported sitting time by season suggested no variation across seasons (Fig. 1).

Correlation between average self-reported sitting time and pedometer-assessed step counts, adjusted for age and sex, was -0.144 (95\% CI $-0.280,0.002)$. We observed a 26.6 \% (95 \% CI 18.2, 39.1) higher step count value during spring/summer but the spring/summer- fall/winter difference in sitting time was inconclusive $(7.1 \%$; $95 \%$ CI -4.4, 18.7) (Fig. 2).

Univariate associations with sitting time were observed for socio-demographic factors (age, immigrant status, university education, annual household income, job status) and for clinical factors (waist circumference and depressed mood) (Table 1). In the subgroup of participants with information on vehicle access $(n=87)$, having regular access to a vehicle was associated with almost a halfhour increase in daily sitting time $(92.0 \mathrm{~min} /$ day, $95 \%$ CrI 6.7, 179.2) compared to those not having access to a vehicle. Because BMI and waist circumference were strongly correlated $(r=0.83,95 \%$ CI $0.79,0.87)$, only waist circumference was included in the final multivariate model. Vehicle access was not included in the final multivariate model because data were only available for a subsample. The impact of clinical factors decreased when integrated into a full multivariate model. In the multivariate model, being an immigrant was associated with less sitting time $(-55.7,95 \%$ CrI: $-99.9,-11.3)$ and having completed a university degree was associated with more (54.8, 95 \% CrI: 10.0, 100.3) (Table 2).

\section{Discussion}

In our study sample of adults with type 2 diabetes, an average of over $5 \mathrm{~h}$ of sitting time/day was reported. While university-educated individuals reported sitting

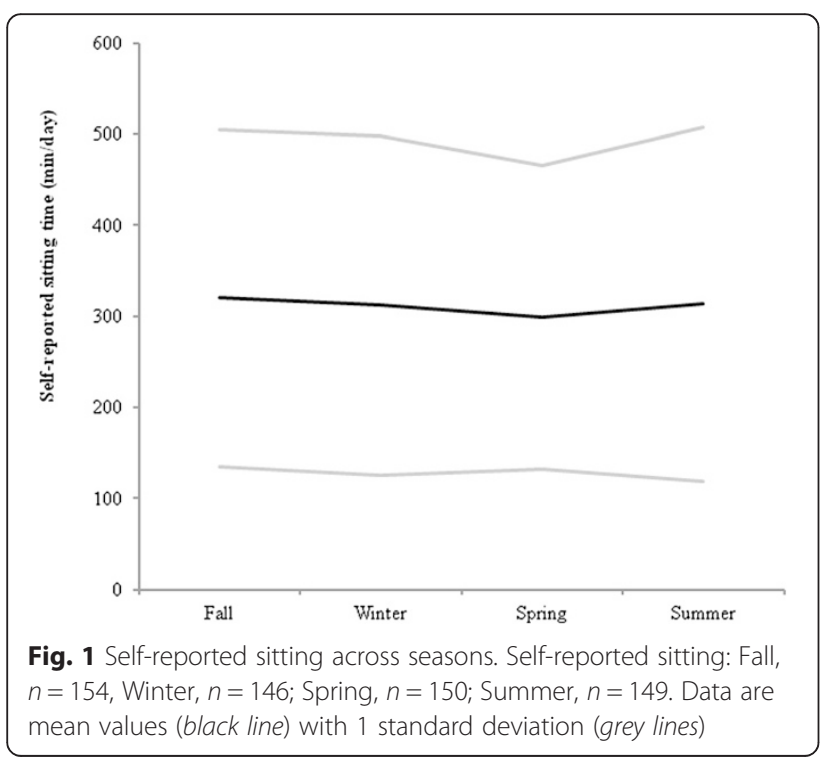

$55 \mathrm{~min} /$ day more than other individuals, immigrants reported $56 \mathrm{~min} /$ day less than non-immigrants. There was no seasonal variation in sitting time observed. Further, there was no important association between self-reported sitting time and pedometer-assessed step counts. Therefore, strategies aimed at reducing sitting time appear to require targeting a subgroup that differs somewhat from those with lower step counts. The absence of association differed from a previous study among young adult workers among whom there was a negative association between self-reported sitting and walking [22].

Studies in other groups (e.g. Canadian Community Health Survey, Epic Norfolk) [23, 24] have largely focused on television and screen viewing as metrics of sedentary time. Older age, being unemployed or retired, higher body mass index and depressive symptomology have been shown to be associated with more television viewing [25]. However, increased sitting time may be related to work and transport-related factors, not just television viewing; less is known about overall sitting time and its correlates [25]. The amount of sitting time reported in our type 2 diabetes cohort is similar to that reported in population-based multi-country studies using self-reported measures [12, 13]. We further assessed for differences across seasons. In contrast to step counts (758 fewer steps/day in fall/winter compared to spring/summer) [4], we demonstrated that sitting time did not vary importantly across seasons. Thus, the seasonal reduction in steps does not appear to directly lead to an increase in overall sitting time. One possibility that we considered was that the absence of seasonal variation in sitting time was the result of using self-report methods. However, with respect to physical activity, our analyses demonstrated seasonal variation not only for objectivelyassessed step counts but also for self-reported walking, as captured through the IPAQ-SV. Both measures of walking showed important increases during spring/summer compared to fall/winter.

Accordingly, correlates of reported sitting time differ from those associated with steps [26, 27]. Indeed, it has been demonstrated that interventions aimed at increasing physical activity do not necessarily reduce sitting time [28]. A recent systematic review also reported a weak to moderate inverse relationship between sedentary behaviors in general and physical activity [29]. Further, even among those achieving the recommended $150 \mathrm{~min} /$ week of physical activity, sitting more than $8 \mathrm{~h} /$ day still confers an elevated risk of all-cause mortality [30]. Thus, it appears that both low levels of physical inactivity and high amounts of sitting time need to be separately targeted to achieve maximum health benefit [31].

Given that the IPAQ-SV queries overall sitting time on weekdays, a large part of self-reported sitting time may be work-related. High educational level is often associated with professional office setting environments (i.e., 


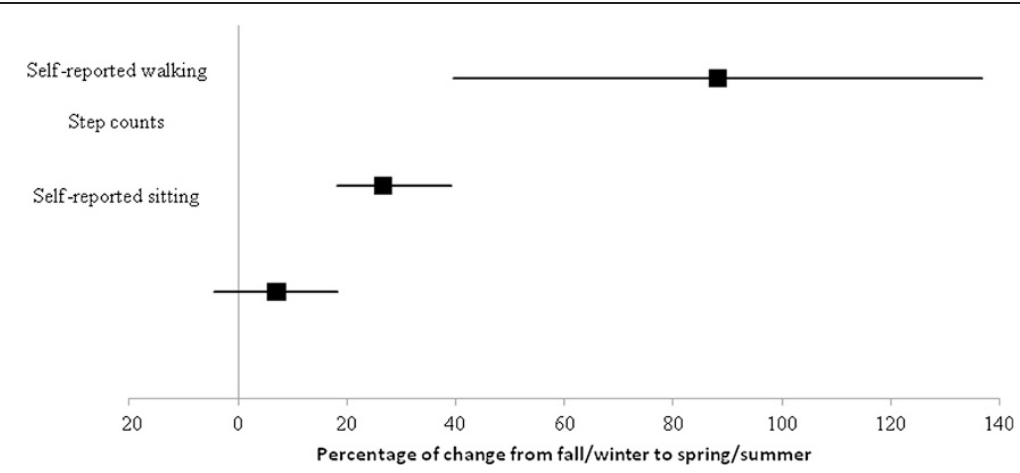

Fig. 2 Behaviours' change between seasons. Changes between seasons were available for 142 individuals. Data are mean with $95 \%$ confidence intervals

so-called "white-collar" jobs). These are in contrast to "blue-collar" jobs that are generally more physically demanding. The higher time spent sitting observed among people with a university degree may thus reflect the type of job they have. Consistent with our findings, low education status was negatively related to self-reported weekday sitting time among young adult Australian women (unstandardized B -1.23, 95 \% CI -1.38, -1.09) [32]. Similarly, in this Australian study, people with a blue-collar occupation and those working less than $35 \mathrm{~h}$ per week reported sitting less during the week [32]. However, given that $43 \%$ of our cohort was retired or unemployed, having a university degree in some individuals was likely related to non work-related activities that increased sitting time.

Immigrants demonstrated lower sitting time, even after adjustment for work status, educational level and age. Recent immigrants are twice as likely as Canadian-born Montréal residents to use public transit in their daily commute [33]. Compared to Canadian born individuals, immigrants are more likely to work in processing and manufacturing industries and sales and service occupations [34]. These factors may in part explain the lower daily sitting time observed in this group of individuals.

Although having a car was associated with increased sitting time $(92.0 \mathrm{~min} /$ day, $95 \% \mathrm{CrI} 6.7,179.2)$ in univariate analysis, we did not include this variable in the multivariate analysis because data were available for only $44 \%$ of our sample. This one and half hour increase in self-reported sitting time in this subgroup is nonetheless substantial. In addition to university education and not being an immigrant, in univariate analyses, younger age, currently working, high annual income, depressed mood, and higher waist circumference were all associated with higher sitting time; further, male sex, not being in a married or common-law relationship, and shorter diabetes duration showed trends towards associations with more sitting time. In multivariate analyses, associations of sitting time with university education and not being an immigrant were confirmed. There were trends suggestive of associations with younger age and currently working, but these were not conclusive.

The IPAQ is the most widely used instrument to assess sitting at the population level and thus its use facilitates comparison with other studies. We acknowledge that the IPAQ-SV does not distinguish between the different domains of sitting, such as work-related, transportation, home-related, and leisure-time nor does it provide information on extended sitting time versus sitting occurring in short bouts. Moreover it queries only the information about week days in contrast to the IPAQ long version.

Table 2 Multivariate longitudinal hierarchical linear regression estimates between the predictors of interest and daily sitting time in minutes $(n=191)$

\begin{tabular}{ll}
\hline & Increment in minutes of daily sitting time $(95 \%$ Crl) \\
\hline Sex (men) & $22.5(-23.3,68.3)$ \\
Currently working & $-2.4(-5.0,0.0)$ \\
University education & $42.9(-9.1,93.4)$ \\
Immigrant & $54.8(10.0,100.3)$ \\
Waist circumference, per cm & $-55.7(-99.9,-11.3)$ \\
Absence of depressed mood & $1.1(-0.7,2.9)$ \\
Daily steps, per step & $-11.1(-61.2,38.6)$ \\
\hline
\end{tabular}


Both the short and long versions are subject to recall bias in contrast to objective accelerometry measures. Healy and colleagues, in a review of validation studies, reported low-to-moderate correlations between self-reported sitting and accelerometer-assessed sedentary time. The IPAQ-SV tends to underestimate sitting time when compared to accelerometer-assessed sedentary time [35]. While this may partly be the result of recall bias, it may also be due to the fact that IPAQ specifically queries sitting, a subset of sedentary behaviour, while accelerometry captures overall sedentary behaviors and not specifically sitting. The French version of the IPAQ long form has been validated in a cohort of diabetes patients $(n=143 ; 60.9$ SD 10.5 years of age) and showed similar results to non-clinical populations [36]. Despite the limitations of using the IPAQ-SV in our study, it does allow us to specifically focus on the issue of sitting and its distinction from low step counts in type 2 diabetes.

\section{Conclusion}

Our analyses did not demonstrate a relationship between sitting time and clinical variables; however, there is evidence from other larger studies of such a relationship $[9,10]$. In our study, adults with type 2 diabetes reported an average sitting time similar to the general population that was without variation across seasons. Socio-demographic factors, such as having completed a university degree and being a non-immigrant, were linked to self-reported sitting time. There was no correlation between sitting time and step counts. Therefore, high sitting time and low step counts are behaviours that may need to be independently targeted. Interventions to reduce sitting time could include home and work environments designed to allow tasks to be completing from a standing position (e.g., standing desks, standingonly office meetings/gatherings or standing up every hour to break prolonged sitting) [37].

\section{Abbreviations}

BMI: Body mass index; CES-D: Center for Epidemiologic Studies - Depression scale; Cl: Confidence interval; Crl: Credible interval; IPAQ-SV: International Physical Activity Questionnaire - Short version; IQR: Interquartile range; SD: Standard deviation.

\section{Competing interests}

The authors declare that they have no competing interests.

\section{Authors' contributions}

ASB analysed and interpreted the data and wrote the manuscript; SH analysed and interpreted the data and reviewed the manuscript; $L J$ analysed the data and reviewed the manuscript; KD designed the study, interpreted the data and wrote the manuscript. All authors read and approved the final manuscript.

\section{Acknowledgements}

The authors thank Patrick Bélisle and Debbie Chan for their assistance with programming and data management. Data collection for this study was funded by an operating grant from the Canadian Institutes of Health Research (CIHR) (KD: MOP-79275). Analyses were supported by an operating grant from the Heart and Stroke Foundation of Canada (KD). ASB is supported by a Heart and Stroke Foundation post-doctoral award. SH is supported by a CIHR Doctoral Research
Award. KD is supported through a Fonds de Recherche en Santé du Québec Senior Clinician Scientist Award.

Received: 5 January 2015 Accepted: 24 July 2015

Published online: 19 August 2015

\section{References}

1. Lanningham-Foster L, Nysse LJ, Levine JA. Labor saved, calories lost: the energetic impact of domestic labor-saving devices. Obes Res. 2003;11(10):1178-81. doi:10.1038/oby.2003.162.

2. Tudor-Locke CE, Bell RC, Myers AM, Harris SB, Lauzon N, Rodger NW. Pedometer-determined ambulatory activity in individuals with type 2 diabetes. Diabetes Res Clin Pract. 2002;55:191-9.

3. Tudor-Locke C, Bassett Jr DR. How many steps/day are enough? Preliminary pedometer indices for public health. Sports Med. 2004;34(1):1-8.

4. Dasgupta K, Joseph L, Pilote L, Strachan I, Sigal RJ, Chan C. Daily steps are low year-round and dip lower in fall/winter: findings from a longitudinal diabetes cohort. Cardiovasc Diabetol. 2010;9:81. doi:10.1186/1475-2840-9-81.

5. Manjoo P, Joseph L, Pilote L, Dasgupta K. Sex differences in step countblood pressure association: a preliminary study in type 2 diabetes. PLoS One. 2010;5(11), e14086. doi:10.1371/journal.pone.0014086.

6. Manjoo P, Joseph L, Dasgupta K. Abdominal adiposity and daily step counts as determinants of glycemic control in a cohort of patients with type 2 diabetes mellitus. Nutr Diabetes. 2012;2, e25. doi:10.1038/nutd.2011.22.

7. Bravata DM, Smith-Spangler C, Sundaram V, Gienger AL, Lin N, Lewis R, et al. Using pedometers to increase physical activity and improve health. A systematic review. JAMA. 2007;289:2296-304.

8. Yates T, Haffner SM, Schulte PJ, Thomas L, Huffman KM, Bales CW, et al. Association between change in daily ambulatory activity and cardiovascular events in people with impaired glucose tolerance (NAVIGATOR trial): a cohort analysis. Lancet. 2014;383(9922):1059-66. doi:10.1016/S01406736(13)62061-9.

9. Wilmot EG, Edwardson CL, Achana FA, Davies MJ, Gorely T, Gray LJ, et al. Sedentary time in adults and the association with diabetes, cardiovascular disease and death: systematic review and meta-analysis. Diabetologia. 2012;55(11):2895-905. doi:10.1007/s00125-012-2677-z.

10. Bjork Petersen C, Bauman A, Gronbaek M, Wulff Helge J, Thygesen LC, Tolstrup JS. Total sitting time and risk of myocardial infarction, coronary heart disease and all-cause mortality in a prospective cohort of Danish adults. Int J Behav Nutr Phys Activ. 2014;11:13. doi:10.1186/1479-5868-11-13.

11. Matthews CE, George SM, Moore SC, Bowles HR, Blair A, Park Y, et al. Amount of time spent in sedentary behaviors and cause-specific mortality in US adults. Am J Clin Nutr. 2012;95(2):437-45. doi:10.3945/ajen.111.019620.

12. Bauman A, Ainsworth BE, Sallis JF, Hagstromer M, Craig CL, Bull FC, et al. The descriptive epidemiology of sitting. A 20-country comparison using the International Physical Activity Questionnaire (IPAQ). Am J Prev Med. 2011;41(2):228-35. doi:10.1016/j.amepre.2011.05.003.

13. Bennie JA, Chau JY, van der Ploeg HP, Stamatakis E, Do A, Bauman A. The prevalence and correlates of sitting in European adults - a comparison of 32 Eurobarometer-participating countries. Int J Behav Nutr Phys Activ. 2013;10:107. doi:10.1186/1479-5868-10-107.

14. Booth GL, Kapral MK, Fung K, Tu JV. Relation between age and cardiovascular disease in men and women with diabetes compared with non-diabetic people: a population-based retrospective cohort study. Lancet. 2006;368(9529):29-36.

15. Dasgupta K, Chan C, Da Costa D, Pilote L, De Civita M, Ross N, et al. Walking behaviour and glycemic control in type 2 diabetes: seasonal and gender differences-study design and methods. Cardiovasc Diabetol. 2007:6:1. doi:10.1186/1475-2840-6-1.

16. Craig $C L$, Marshall AL, Sjostrom M, Bauman AE, Booth ML, Ainsworth BE, et al. International physical activity questionnaire: 12-country reliability and validity. Med Sci Sports Exerc. 2003;35(8):1381-95. doi:10.1249/ 01.MSS.0000078924.61453.FB.

17. Rosenberg DE, Bull FC, Marshall AL, Sallis JF, Bauman AE. Assessment of sedentary behavior with the International Physical Activity Questionnaire. J Phys Act Health. 2008;5 Suppl 1:S30-44.

18. Kurtze N, Rangul V, Hustvedt BE. Reliability and validity of the international physical activity questionnaire in the Nord-Trondelag health study (HUNT) population of men. BMC Med Res Methodol. 2008;8:63. doi:10.1186/14712288-8-63. 
19. Dyrstad SM, Hansen BH, Holme IM, Anderssen SA. Comparison of selfreported versus accelerometer-measured physical activity. Med Sci Sports Exerc. 2014;46(1):99-106. doi:10.1249/MSS.0b013e3182a0595f.

20. Patterson E. IPAQ scoring protocol. 2010. https://sites.google.com/site/theipaq/ scoring-protocol. Accessed 15 August 2015.

21. Radloff LS. The CES-D Scale: A Self-Report Depression Scale for Research in the General Population. Appl Psychol Meas. 1977;1(3):385-401.

22. Miller $\mathrm{R}$, Brown W. Steps and sitting in a working population. Int J Behav Med. 2004;11(4):219-24. doi:10.1207/s15327558ijbm1104_5.

23. Wijndaele K, Brage S, Besson H, Khaw KT, Sharp SJ, Luben R, et al. Television viewing and incident cardiovascular disease: prospective associations and mediation analysis in the EPIC Norfolk Study. PLoS One. 2011;6(5):e20058. doi:10.1371/journal.pone.0020058.

24. Shields M, Tremblay MS. Screen time among Canadian adults: a profile. Health reports/Statistics Canada, Canadian Centre for Health Information = Rapports sur la sante/Statistique Canada. Centre canadien d'information sur la sante. 2008;19(2):31-43.

25. Rhodes RE, Mark RS, Temmel CP. Adult sedentary behavior: a systematic review. Am J Prev Med. 2012;42(3):e3-28. doi:10.1016/j.amepre.2011.10.020.

26. Hajna S, Ross N, Dasgupta K. Individual and socio-environmental determinants of walking in adults with type 2 diabetes. Diabetes. 2012;61(S1):A381.

27. Hajna S, Ross N, Dasgupta K. Dog ownership is associated with higher daily step counts in the fall and winter in adults living with type 2 diabetes. Can J Diabetes. 2013;37:s242.

28. Finni T, Haakana P, Pesola AJ, Pullinen T. Exercise for fitness does not decrease the muscular inactivity time during normal daily life. Scand J Med Sci Sports. 2014;24(1):211-9. doi:10.1111/j.1600-0838.2012.01456.x.

29. Mansoubi M, Pearson N, Biddle SJ, Clemes S. The relationship between sedentary behaviour and physical activity in adults: A systematic review. Prev Med. 2014;69C:28-35. doi:10.1016/j.ypmed.2014.08.028.

30. van der Ploeg HP, Chey T, Korda RJ, Banks E, Bauman A. Sitting time and all-cause mortality risk in 222497 Australian adults. Arch Intern Med. 2012;172(6):494-500. doi:10.1001/archinternmed.2011.2174.

31. Bell JA, Hamer M, Batty GD, Singh-Manoux A, Sabia S, Kivimaki M. Combined effect of physical activity and leisure time sitting on long-term risk of incident obesity and metabolic risk factor clustering. Diabetologia. 2014;57(10):2048-56. doi:10.1007/s00125-014-3323-8.

32. Uijtdewilligen L, Twisk JW, Singh AS, Chinapaw MJ, van Mechelen W, Brown WJ. Biological, socio-demographic, work and lifestyle determinants of sitting in young adult women: a prospective cohort study. Int J Behav Nutr Phys Activ. 2014;11:7. doi:10.1186/1479-5868-11-7.

33. Heisz A. Canada's global cities: Socio-economic conditions in Montréal, Toronto and Vancouver. Ottawa: Minister of Industry; 2006.

34. Badets J, Howatson-Leo L. Recent immigrants in the workforce. Ottawa: Statistics Canada; 1999. p. 16-23.

35. Healy GN, Clark BK, Winkler EA, Gardiner PA, Brown WJ, Matthews CE. Measurement of adults' sedentary time in population-based studies. Am J Prev Med. 2011;41(2):216-27. doi:10.1016/j.amepre.2011.05.005.

36. Criniere L, Lhommet $C$, Caille A, Giraudeau B, Lecomte P, Couet $C$, et al. Reproducibility and validity of the French version of the long international physical activity questionnaire in patients with type 2 diabetes. J Phys Act Health. 2011;8(6):858-65.

37. Chau JY, Daley M, Srinivasan A, Dunn S, Bauman AE, van der Ploeg HP Desk-based workers' perspectives on using sit-stand workstations: a qualitative analysis of the Stand@Work study. BMC Public Health. 2014:14(1):752. doi:10.1186/1471-2458-14-752.

\section{Submit your next manuscript to BioMed Central and take full advantage of:}

- Convenient online submission

- Thorough peer review

- No space constraints or color figure charges

- Immediate publication on acceptance

- Inclusion in PubMed, CAS, Scopus and Google Scholar

- Research which is freely available for redistribution 